\title{
Hemorrhage after laser-cut covered self-expandable metal stent removal
}

Endoscopic covered self-expandable metal stent (CSEMS) placement is widely used for distal malignant biliary obstruction (MBO) as the stent prevents ingrowth and is easily removed [1,2]. The usefulness of the laser-cut CSEMS (LC-CSEMS) and its endoscopic removal have been reported [3-5]; there have been no previous reports of complications related to LC-CSEMS removal. We here report a case of hemorrhage following LC-CSEMS removal.

An 83-year-old woman was referred for cholangitis. She had undergone endoscopic LC-CSEMS (X-Suit NIR covered biliary metal stent; Olympus Medical Systems, Tokyo, Japan) placement 7 months previously for unresectable pancreatic cancer. Endoscopic retrograde cholangiopancreatography (ERCP) was performed because recurrent biliary obstruction was suspected ( $\mathbf{F i g . 1}$; - Video 1). Cholangiography revealed defects suggestive of sludge ( $\mathbf{F i g . 2}$ ). We therefore decided to remove the LCCSEMS and replace it with a new one.

A snare forceps was used to hold the LCCSEMS, which was moved toward the papilla by pushing, along with clockwise torsion of the endoscope while adjusting the axis of the bile duct [3]. The initial attempt to pull the LC-CSEMS out was effective; however, the snare subsequently could not be released, with the plan having originally been to release it from the LC-CSEMS and reposition it nearer the papilla ( Fig.3a). Consequently, the LC-CSEMS was instead removed by withdrawing the endoscope.

After stent removal, we inserted the endoscope to the papilla and found that a hemorrhage had been induced from the papilla during LC-CSEMS removal ( $\triangleright$ Fig. 3 b). Although biliary cannulation was possible, the hemorrhage disturbed the endoscopic view, so re-insertion of the new LC-CSEMS was performed under fluoroscopic guidance. The LC-CSEMS suffers from minimal stent shortening because of the laser-cut structure; there-
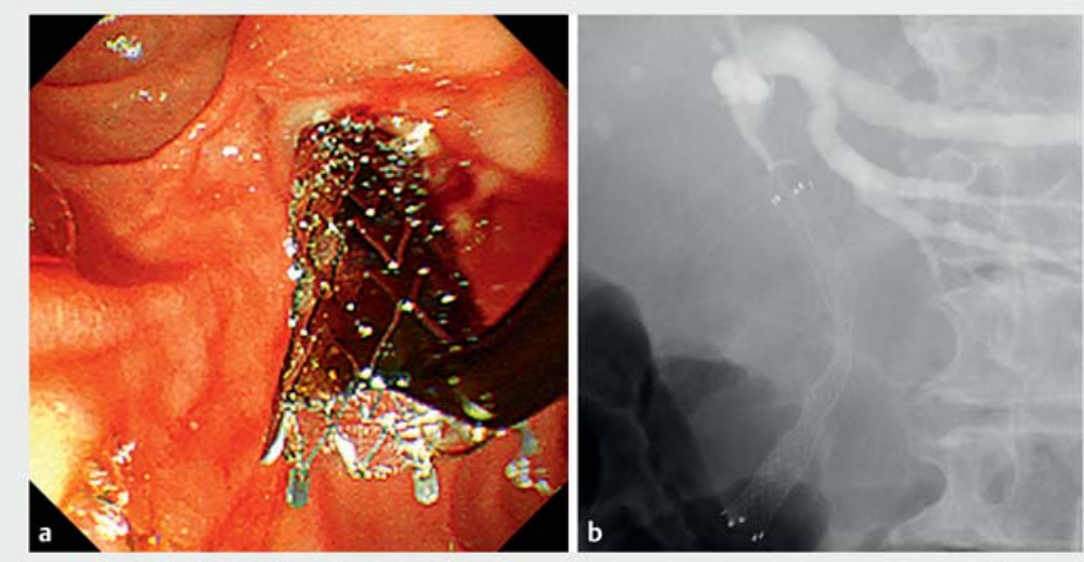

- Fig. 1 Endoscopic retrograde cholangiopancreatography findings showing: a the endoscopic appearance of the successfully placed laser-cut covered self-expandable metal stent (LC-CSEMS) for a patient with unresectable pancreatic cancer; $\mathbf{b}$ the fluoroscopic appearance of the successfully placed LC-CSEMS.
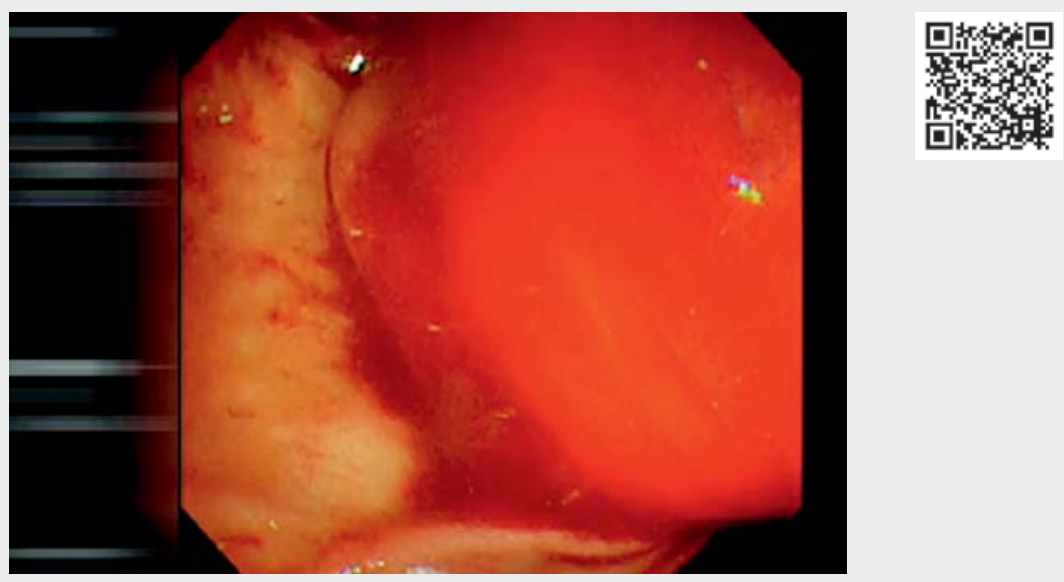

$\checkmark$ Video 1 Difficult removal of a laser-cut covered self-expandable metal stent (LCCSEMS) causing hemorrhage from the papilla that was subsequently controlled by insertion of a replacement LC-CSEMS.

fore, it could be placed easily in an accurate position ( $\mathbf{F i g . 4}$ a) and the hemorrhage was finally stopped by compression from the LC-CSEMS (> Fig.4b). Although this hemorrhage was induced by LC-CSEMS removal, it was easily stopped after the LC-CSEMS was replaced.
Endoscopy_UCTN_Code_CPL_1AK_2AD

Competing interests

The authors declare that they have no conflict of interest. 


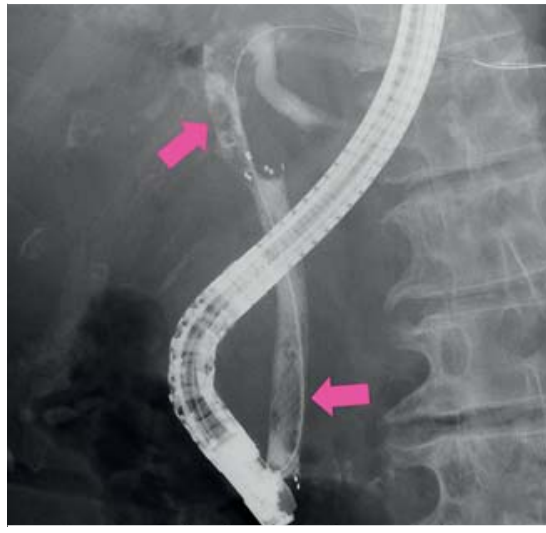

- Fig. 2 Cholangiographic appearance with defects (pink arrows) suggestive of sludge.

\section{Acknowledgments}

We would like to thank Editage (www.editage. com) for English language editing.

The authors

Yuki Tanisaka Masafumi Mizuide, Akashi Fujita ? Tomoya Ogawa, Hiromune Katsuda, Youichi Saito, Shomei Ryozawa

Department of Gastroenterology, Saitama Medical University International Medical Center, Hidaka, Saitama, Japan

\section{Corresponding author}

\section{Yuki Tanisaka, MD, PhD}

Department of Gastroenterology, Saitama Medical University International Medical

Center, 1397-1, Yamane, Hidaka, Saitama 350-1298, Japan

tanisaka1205@gmail.com

\section{References}

[1] Isayama H, Komatsu Y, Tsujino T et al. A prospective randomised study of "covered" versus "uncovered" diamond stents for the management of distal malignant biliary obstruction. Gut 2004; 53: 729-734

[2] Kitano M, Yamashita Y, Tanaka K et al. Covered self-expandable metal stents with an anti-migration system improve patency duration without increased complications compared with uncovered stents for distal biliary obstruction caused by pancreatic carcinoma: a randomized multicenter trial. Am J Gastroenterol 2013; 108: 1713-1722
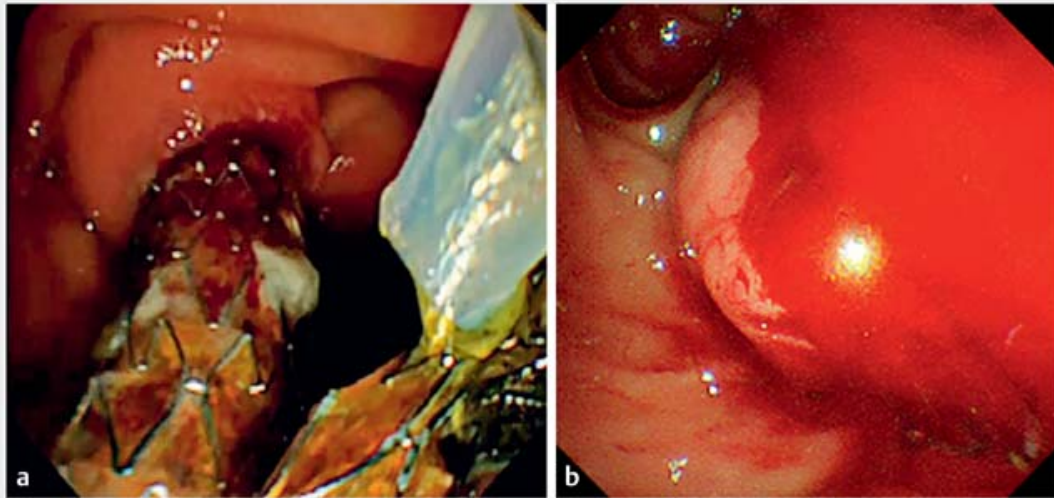

- Fig. 3 Endoscopic views showing: a the snare forceps, which could not be released, holding the partially withdrawn laser-cut covered self-expandable metal stent; $\mathbf{b}$ hemorrhage that was induced during laser-cut covered self-expandable metal stent removal.
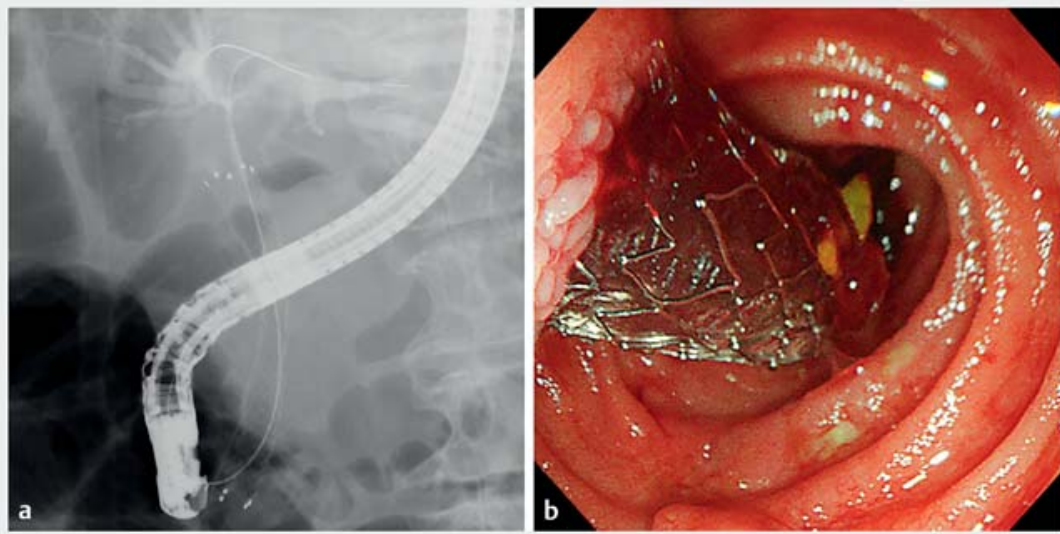

- Fig. 4 Endoscopic retrograde cholangiopancreatography findings showing: a the fluoroscopic appearance of the successfully placed accurately positioned laser-cut covered selfexpandable metal stent (LC-CSEMS); $\mathbf{b}$ the endoscopic appearance with hemostasis successfully achieved by compression from the LC-CSEMS.

[3] Tanisaka Y, Ryozawa S, Kobayashi M et al. Endoscopic removal of laser-cut covered self-expandable metallic biliary stents: A report of six cases. Mol Clin Oncol 2018; 8: 269-273

[4] Marui S, Uza N, Yamazaki H et al. Utility of laser-cut covered self-expandable metal stents for unresectable malignant distal biliary obstruction: a single-center experience. Endoscopy 2020; 52: 664-668

[5] Kin T, Ishii K, Okabe $Y$ et al. Feasibility of biliary stenting to distal malignant biliary obstruction using a novel designed metal stent with duckbill-shaped anti-reflux valve. Dig Endosc 2021; 33: 648-655

\section{Bibliography}

Endoscopy 2022; 54: E378-E379

DOI 10.1055/a-1541-7205

ISSN 0013-726X

published online 9.8.2021

(c) 2021. Thieme. All rights reserved.

Georg Thieme Verlag KG, Rüdigerstraße 14, 70469 Stuttgart, Germany 